\title{
Track Fitting in Belle II: the GENFIT Library and its Performance
}

\author{
Tobias Schlüter ${ }^{* \dagger}$ for the Belle II Software Group \\ Ludwig-Maximilians-Universität München, Germany \\ E-mail: tobias.schlueterephysik.uni-muenchen.de
}

\begin{abstract}
We discuss track fitting as implemented in the Belle II experiment currently under construction at KEK in Tsukuba, Japan. Track fitting takes place at the interface of physics analysis and detector data, is essential to the tasks of detector calibration and alignment, and it also is an integral part of Belle II's high-level trigger. To address this variety of tasks, the track-fitting software, initially based on the GENFIT library, underwent significant redesign. The revised version aims at being experiment-independent and is now the default track-fitting software in the Belle II, PANDA, and SHiP experiments. It implements a variety of track-fitting algorithms, provides a data storage model which allows storage at different levels of detail and provides high-level operations on tracks such as combinations of tracks from different subdetectors. It provides visualization, and supports all commonly employed types of tracking detectors. It provides a Runge-Kutta type track extrapolation code with handling of energy loss and multiple scattering. It interfaces to the commonly used Millipede II software for detector calibration and alignment, and to the experiment-independent vertexing library RAVE.
\end{abstract}

PACS: $07.05 . \mathrm{Rm}, 29.40 . \mathrm{Gx}, 29.85 . \mathrm{Ca}$

The European Physical Society Conference on High Energy Physics

22-29 July 2015

Vienna, Austria

\footnotetext{
* Speaker.

†This research was supported by the DFG cluster of excellence 'Origin and Structure of the Universe' and under BMBF Contracts 05H12WM8 and 05H15WMKBA.
} 


\section{Introduction}

Track fitting concerns itself with the problem of combining detector hits provided by a trackfinding procedure in order to extract precise information on the trajectories of particles in particlephysics experiments. The fitted tracks are then used for physics data analysis (vertex reconstruction) but also for detector optimization (alignment, calibration) as well as in event selection and processing (quality indicators, outlier rejection). The task of track fitting is tightly coupled to a trajectory model, i.e. an extrapolation code. Previous considerations of the topic lead to the development of the experiment-independent track-fitting library GENFIT [1] which was also adopted by the Belle II collaboration. This software turned out to be too limited for the uses of the Belle II collaboration, and thus it was essentially rewritten. This new version [2] still aims to be generic and experiment-independent. It is written in $\mathrm{C}++03$ and licensed under a Free Software license (LGPL) and it is the default track-fitting software not only of the Belle II collaboration, but also of the PANDA and SHiP [3] collaborations (and perhaps others that we are not aware of). This contribution discusses our experience in Belle II.

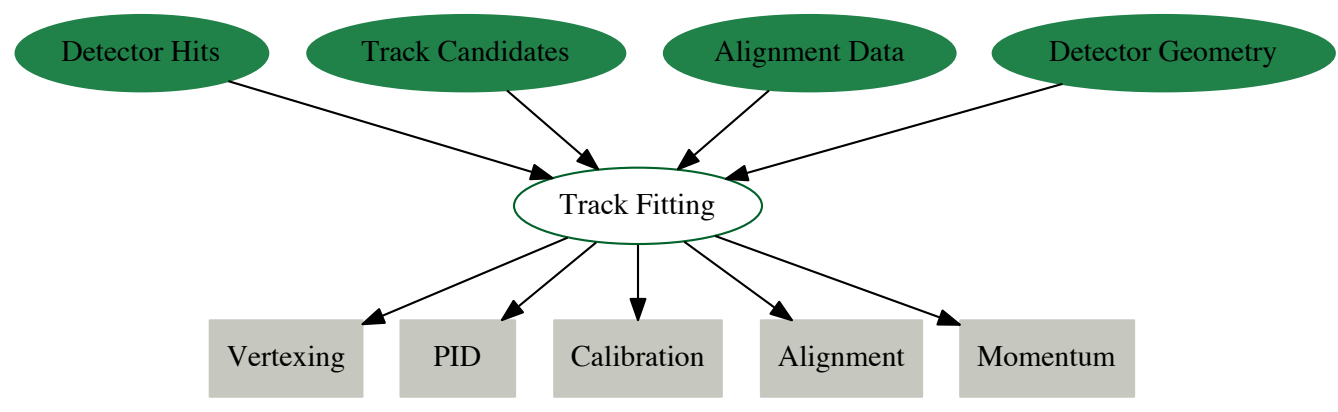

Figure 1: Track fitting as convergence point of various data and threads of data reconstruction

Inputs for track fitting are detector hits together with possible trajectories (the track candidates) and information on the detector geometry and its finer details, such as alignment data. The fit provides outputs which are relevant to various tasks such as the subsequent vertexing and particle identification in physics analysis or tasks such as calibration or alignment. In Fig. 1 we illustrate this focal role.

\section{Track Fitting In Belle II}

In Belle II track fitting is both executed online (for data reduction and as part of the high-level trigger) [4] and offline during the preparation of the mDST files used for data analysis. The Belle II experiment has the cylindrical geometry typical of a collider experiment. Its tracking hardware consists of a vertex detector close to the beampipe surrounded by a drift chamber. The vertex detector consists of six layers of silicon detectors. The two innermost layers are of DEPFET type with pixel readout [5], the outer four layers are silicon strip detectors with double-sided read out [6]. 
The drift chamber contains of nine wire superlayers, in an alternating configuration of axial wires (parallel to the magnetic field) and stereo wires [7]. Throughout the tracking volume, the magnetic filed is almost constant up to $\pm 2 \%$. The tracking detectors are surrounded by further detectors for particle identification and neutral particle detection $[8,9]$.

Track finding proceeds separately on the vertex detector (using a cellular automaton together with Hopfield network to resolve overlapping candidates [10]) and the drift chamber (using a global strategy based on Legendre transformation [11] and a local, cellular-automaton-based approach [12]), the track candidates from the subdetectors are either combined based on MC information, or, after fitting, based on the reconstructed parameters at the boundary between the subdetectors. Software is in development to pick up additional hits for trajectories that have hits in both subdetectors but can only be reconstructed independently in one of them, either because they traversed only part of the vertex detector (such as trajectories from $V^{0}$ decays) or because they are slow particles that curl within the first superlayer of the drift chamber which by itself does not provide enough information for complete track reconstruction. The combinatorial Kalman filter algorithm [13] will be used for this purpose.

Data is processed using the same software on the high-level trigger and offline. The software framework organizes data handling by sequentially processing a sequence of data handling modules [14]. The sequence is configured by a steering file written in the Python programming language. The modules read from and write to a common data store whose contents are streamed to disk after processing, thus ensuring persistency. Configuration data is read from a database. Track fitting is performed as part of this sequence. The track-fitting module reads track candidates and detector hits from the data store, feeds them into GENFIT, and the fitted tracks output from GENFIT are copied to the data store. There they are available to subsequent processing. In particular, the track parameters are converted to a helix parameterization, and only this is kept in the final mDST files used for physics analysis.

GENFIT implements a variety of track-fitting algorithms. These include the standard Kalman filter algorithm [15] and the deterministic annealing filter (DAF) [16, 17] as well as the Generalized Broken Lines algorithm (GBL) [18, 19]. These serve different purposes in the experiment. The Kalman filter will be used on the high-level trigger. The default track fitting algorithm in the offline processing will be the DAF, as it is expected to be more robust in the presence of the expected high background levels. GBL is used to interface the Millipede II software used for alignment [20, 21]. For extrapolation, we rely on the extrapolation code of GENFIT which shares the same heritage as the STEP code used by the ATLAS experiment [22, 23]. It is an adaptive Runge-Kutta-Nyström extrapolator of fourth order which semi-analytically updates the transport Jacobians along with the numerical evaluation of the track parameters. Features specifically developed for the low-momentum environment of Belle II include precise handling of energy loss and the calculation of flight-time estimates that take into account energy loss along the track as well as the effects of different mass hypotheses. In order to facilitate vertexing, GENFIT interfaces with the RAVE library [24], a generic vertexing toolkit. Finally, it provides visualization utilities that allow studying all stages of track fitting. In this way, pathological cases can be understood, and it is also a useful utility for teaching. An example illustration is given in Fig. 2. 


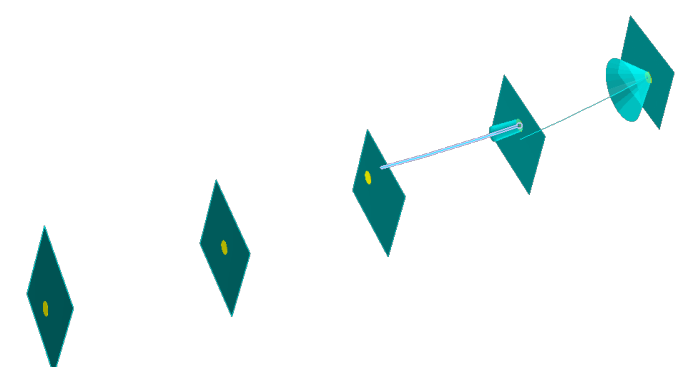

(a) Forward fit after predicting the coordinates on the third plane

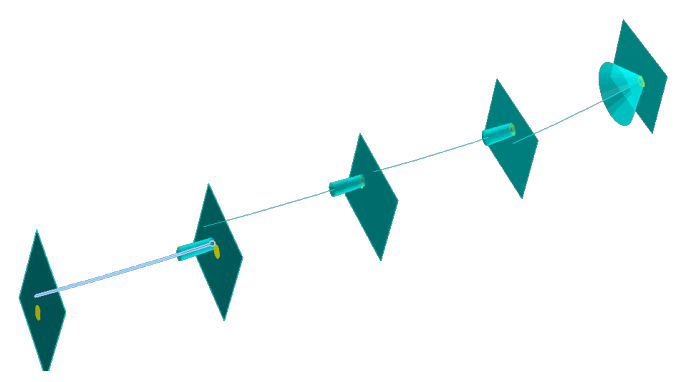

(b) Completed forward fit

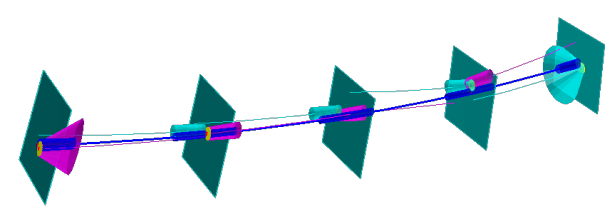

(d) Smoothed track overlayed (blue)

Figure 2: Visualization of the Kalman fit for a sequence of five 2D hits (indicated by yellow ellipses on their respective detector planes). The particle traverses the detectors from right to left. Error estimates for the track parameters are indicated as cones, extrapolations by thin lines. Elements corresponding to the forward fit are drawn in light green-blue, those corresponding to the backward fit in a reddish tone.

\section{Performance in Belle II}

A typical $B \bar{B}$ event in Belle II will have $O(10)$ charged tracks with a transverse momentum distribution peaking below $200 \mathrm{MeV} / c$. The expected momentum distribution is illustrated in Fig. 3 . Further, one of these tracks will typically be a charged Kaon (at these momenta, the heavy Kaon propagates significantly slower than the speed of light, and its energy loss will appreciably exceed that of the pions), and on average one $K_{S}^{0}$ will be present. On a typical desktop PC, using Belle II's default choice of the DAF as track fitting algorithm, a single track is fitted within $20 \mathrm{~ms}$, where the time is dominated by geometry lookups in the Geant 4 geometry. Retaining all track fitting information (amongst others, this includes Jacobians, noise matrices, local track coordinates and covariances at each hit along with the hit information), a single track contains approximately $70 \mathrm{~kb}$ of data. After performing $d E / d x$-based particle PID, only the information at the two extremities of the track is retained, on the inside for vertexing, on the outside for extrapolation to the outer detectors. This cuts the data volume down to $1.3 \mathrm{~kb}$ per track. Finally, for the purpose of physics analysis, only helix parameters are stored. These together with their covariance matrix, and some quality information, amount to 170 bytes per track.

Performance of the Belle II software stack is continuously validated based on Monte Carlo 


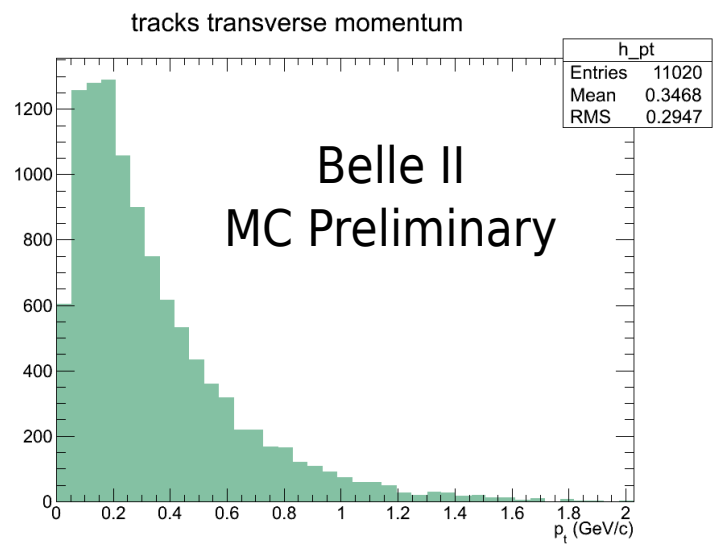

Figure 3: Monte Carlo momentum spectrum of charged particles in Belle II.

data samples. Regressions in reconstruction quality can thus be pinpointed easily. The handling of actual detector data has also been established. For the vertex detector, a dedicated test-beam experiment established the functioning of the complete read-out chain, both online tracking on the high-level trigger and offline processing of data was performed successfully [25]. For the central drift chamber, first results from a cosmic ray test which is taking place at the time of this writing are also encouraging.

\section{References}

[1] C. Höppner et al. "A Novel Generic Framework for Track Fitting in Complex Detector Systems”. In: Nucl.Instrum.Meth. A620 (2010), pp. 518-525. arXiv: 0911.1008 [hep-ex ] .

[2] J. Rauch and T. Schlüter. "GENFIT - a Generic Track-Fitting Toolkit". In: J.Phys.Conf.Ser. 608.1 (2015), p. 012042. arXiv: 1410.3698 [physics.ins-det ]

[3] M. Anelli et al. A facility to Search for Hidden Particles (SHiP) at the CERN SPS. 2015. arXiv: 1504.04956 [physics.ins-det] .

[4] S. Lee et al. "Development of high level trigger software for Belle II at SuperKEKB". In: $J$. Phys. Conf. Ser. 331 (2011), p. 022015.

[5] O. Alonso et al. "DEPFET active pixel detectors for a future linear $e^{+} e^{-}$collider". In: IEEE Trans. Nucl. Sci. 60.2 (Apr. 2013), pp. 1457-1465. arXiv: 1212.2160 [phys ics . ins-det ] .

[6] M. Friedl et al. "The Belle II Silicon Vertex Detector”. In: Nucl.Instrum.Meth. A732 (2013), pp. 83-86.

[7] N. Taniguchi et al. "All-in-one readout electronics for the Belle-II Central Drift Chamber". In: Nucl.Instrum.Meth. A732 (2013), pp. 540-542.

[8] T. Abe et al. Belle II Technical Design Report. Tech. rep. 2010. arXiv: 1011.0352 [physics.ins-det].

[9] I. Adachi. "Status of Belle II and SuperKEKB". In: JINST 9 (2014), p. C07017. 
[10] R. Frühwirth et al. "Track finding in silicon trackers with a small number of layers". In: Nucl. Instrum. Meth. A732 (2013), pp. 95-98.

[11] T. Alexopoulos et al. "Implementation of the Legendre Transform for track segment reconstruction in drift tube chambers". In: Nucl. Instrum. Meth. A592 (2008), pp. 456-462.

[12] O. Frost. "A Local Tracking Algorithm for the Central Drift Chamber of Belle II". MA thesis. Karlsruhe Institute of Technology, 2013.

[13] R. Mankel. "A concurrent track evolution algorithm for pattern recognition in the HERA-B main tracking system”. In: Nucl. Instrum. Meth. A395 (1997), pp. 169-184.

[14] D. Y. Kim. "The software library of the coming Belle II experiment and its simulation package". In: Proceedings, 2013 IEEE Nuclear Science Symposium and Medical Imaging Conference (NSS/MIC 2013). 2013.

[15] R. Frühwirth et al. Data Analysis Techniques for High-Energy Physics. Cambridge University Press, 2000.

[16] R. Frühwirth and A. Strandlie. "Application of adaptive filters to track finding". In: Nucl. Instrum. Meth. A559 (2006), pp. 162-166.

[17] S. Fleischmann. "Track reconstruction in the ATLAS experiment: The deterministic annealing filter". Wuppertal U., 2006.

[18] V. Blobel. "A new fast track-fit algorithm based on broken lines". In: Nucl. Instrum. Meth. A566 (2006), pp. 14-17.

[19] C. Kleinwort. "General Broken Lines as advanced track fitting method". In: Nucl. Instrum. Meth. A673 (2012), pp. 107-110. arXiv: 1201.4320 [physics.ins-det] .

[20] V. Blobel and C. Kleinwort. "A new method for the high precision alignment of track detectors". In: Advanced statistical techniques in particle physics. Proceedings, Conference, Durham, UK, March 18-22, 2002. 2002. arXiv: hep-ex/0208021.

[21] T. Bilka. "Alignment of the Belle II Vertex Detector". In: Proceedings, 23rd International Workshop on Vertex Detectors (Vertex 2014). PoS (Vertex2014) 048.

[22] E. Lund et al. "Track parameter propagation through the application of a new adaptive Runge-Kutta-Nystroem method in the ATLAS experiment”. In: JINST 4 (2009), P04001.

[23] E. Lund et al. "Transport of covariance matrices in the inhomogeneous magnetic field of the ATLAS experiment by the application of a semi-analytical method". In: JINST 4 (2009), P04016.

[24] W. Waltenberger. "RAVE: A detector-independent toolkit to reconstruct vertices". In: IEEE Trans. Nucl. Sci. 58 (2011), pp. 434-444.

[25] T. Bilka et al. "Demonstrator of the Belle II Online Tracking and Pixel Data Reduction on the High Level Trigger System”. In: IEEE Trans. Nucl. Sci. 62.3 (June 2015), pp. 11551161. ISSN: 0018-9499. arXiv: 1406.4955 [physics.ins-det] . 